\title{
Moving window analysis links landscape-scale resource utilization to habitat suitability models of feral pigs in northern Australia
}

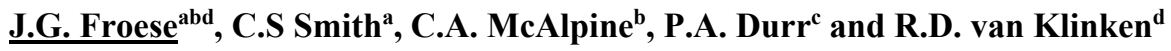 \\ ${ }^{a}$ School of Agriculture \& Food Sciences, The University of Queensland \\ ${ }^{b}$ School of Geography, Planning \& Environmental Management, The University of Queensland \\ ${ }^{c}$ CSIRO Health \& Biosecurity Flagship, Geelong, Victoria \\ ${ }^{d}$ CSIRO Health \& Biosecurity Flagship, Dutton Park, Queensland \\ Email: jens.froese@uq.net.au
}

\begin{abstract}
Habitat suitability (or species distribution) models have been widely used to inform management decisions about species conservation, resource management or invasion control. We developed habitat suitability models of feral pigs across tropical northern Australia to enable more effective management of their negative impacts. However, when investigating wildlife-habitat relationships of highly mobile animals such as feral pigs, home range behaviour must be accounted for as these species can utilize scattered resources and fulfil their living and breeding requirements at different locations within the wider landscape that is accessible to them.
\end{abstract}

We developed a novel approach for incorporating landscape-scale utilization of resources and other habitat requirements into habitat suitability models of feral pigs via moving window analysis. Modelling followed three steps: (1) model pixel values for each of four key habitat requirements (water and food resources as well as protection from heat stress and disturbance) using probabilistic Bayesian networks that combine spatially-explicit explanatory variables, (2) transform them into landscape values via moving window analysis, and (3) model habitat suitability using a Bayesian network that combines all landscape values. Models were implemented in Norsys Netica 5.12 and AgenaRisk 6.1 software and calibrated using expert elicitation. Moving window analyses were implemented in the $R$ 'raster' package and ESRI ArcGIS 10.2 software.

Here, we present expert-elicited response functions describing the relationship between landscape-scale patterns of each habitat requirement and its sufficiency to sustain breeding in feral pigs. Two types of response mechanisms were described by each expert: a distance-dependent one where the sufficiency of a requirement diminishes with increasing distance, and a composition-dependent one where the sufficiency of a requirement diminishes with decreasing abundance. These functions were used to parameterize moving window analyses and calculate distance-dependent, composition-dependent and combined distance/composition-dependent requirement landscape values. Experts described three general shapes of response curve: linear decay, where sufficiency diminishes steadily with increasing distance/decreasing abundance; exponential decay, where sufficiency is strongly affected by initial increases in distance or decreases in abundance; and inverted exponential decay, where sufficiency diminishes little initially but strongly at large distances or low levels of abundance. Experts displayed considerable agreement when describing some responses but differed widely with others. Expert assumptions about the characteristic scale of the response, and hence the size of the moving window, also varied considerably.

We schematically illustrate that expert assumptions about the characteristic scale and mechanism of resource utilization had a considerable effect on computed sufficiency: Assuming distance-dependence dramatically increased sufficiency of a requirement. This effect became more pronounced at larger characteristic scales of resource utilization. Assuming composition- or combined distance/composition-dependence consolidated computed sufficiency to larger, more continuous resource aggregations and discounted utilization of isolated or scattered resources. These effects were equally more pronounced at larger characteristic scales. Hence, these assumptions require careful consideration when using them as an input into habitat suitability models and their effect on model performance must be validated. Our approach could readily be applied to other mobile species that respond to conditions at the landscape scale.

Keywords: Species distribution model, spatial analysis, landscape ecology, home range behaviour, wildlife-habitat relationships 
Froese et al., Moving window analysis links landscape-scale resource utilization to habitat suitability models of feral pigs in northern Australia

\section{INTRODUCTION}

Habitat suitability (or species distribution) models have been widely used to inform management decisions about species conservation, resource management or invasion control. In Australia, feral pigs (Sus scrofa), Family Suidae (order Artiodactyla), are highly successful invaders, causing a multitude of negative impacts on agricultural production, the environment and human and animal health (Bengsen et al. 2014). Effective management of these impacts at the regional scale requires spatially explicit understanding of feral pig populations beyond localized surveys. To address this knowledge gap, we developed habitat suitability models of feral pigs across tropical northern Australia.

Habitat suitability modelling of wildlife species such as feral pigs is complicated by the issue of mobility (Guisan \& Thuiller 2005). Mobile animals are able to utilize scattered resources and fulfil their living and breeding requirements at different locations within the wider accessible landscape. Here, as in the remainder of this paper, the terms landscape and landscape-scale thus refer to a neighbourhood in which species utilize resources and respond to other habitat conditions (Wiens 1989). This neighbourhood can also be related to the home range concept in animal ecology (Burt 1943).

Two main approaches have been suggested to account for landscape-scale mobility and home range behaviour in habitat suitability models: (a) increase the spatial grain at which predictor variables are measured or (b) use focal predictors that summarize information contained within an accessible neighbourhood (Guisan \& Thuiller 2005). Approach (a) has been implemented in previous modelling of feral pig distributions (Cowled et al. 2009) and disease spread (Doran \& Laffan 2005) in Australia. However, in these models each large-grained pixel is treated separately and resource utilization within home ranges that overlap two or more pixels cannot be captured. Furthermore, only approach (b) can preserve fine-grained detail in predictor variables and consider spatial patterns, i.e. the abundance and arrangement of resources.

Approach (b), also termed moving window analysis, has been applied to modelling habitat suitability of other large wildlife species (Dijak et al. 2007). It requires two parameters that relate measurable patterns to ecological processes: the moving window size, which represents the characteristic scale at which species respond to habitat conditions, e.g. the daily home range size (Wiens 1989); and the analysis metric, which represents the underlying mechanism of the species response. Three general mechanisms can be envisaged. Utilization of resources and other habitat requirements could be distance-dependent where sufficiency diminishes with increasing distance, composition-dependent where sufficiency diminishes with decreasing abundance, or configuration-dependent where sufficiency varies with their arrangement and positioning in the landscape (Martin \& Fahrig 2012, McGarigal \& Marks 1995). Yet, empirical data on the characteristic scale and mechanism of species responses to habitat conditions is often limited (Holland et al. 2004).

In this paper, we describe an approach for incorporating landscape-scale utilization of resources and other habitat requirements into habitat suitability models via moving window analysis. Analysis parameters were elicited from experts based on their field knowledge of feral pigs' response to habitat conditions. We schematically illustrate and discuss how different expert assumptions about the characteristic scale and mechanism of the response influenced the computed sufficiency of feral pigs' habitat requirements.

\section{METHODS}

Habitat suitability was defined in terms of the ability of feral pigs to breed and persist. We followed the conceptual framework in Smith et al. (2007), where habitat suitability depends on a set of key habitat requirements representing resources or other habitat conditions such as limiting factors or anthropogenic influences; each habitat requirement is influenced by several spatially-explicit explanatory variables.

Models were implemented as probabilistic Bayesian networks in Norsys Netica 5.12 and AgenaRisk 6.1 software and calibrated during an iterative expert elicitation process (Marcot 2006). Remotely sensed or mapped spatial data layers were rasterized and resampled to a common extent and resolution (100m) and then reclassified to best represent explanatory variables using the $R$ 'raster' package (R Core Team 2015, Hijmans 2015) and ESRI ArcGIS 10.2 software. Modelling followed three steps: (1) model pixel values for each habitat requirement using Bayesian networks that combine spatially-explicit explanatory variables, (2) transform them into landscape values via moving window analysis, and (3) model a habitat suitability index using a Bayesian network combining all landscape values.

Pixel values $x_{r}$ described the presence and quality of each habitat requirement $r$, conditional on the values of its explanatory variables. Requirement quality was discretised into five states, each assigned with a numerical value range (from 0-20 for the worst state to 80-100 for the best state). $x_{r}$ was then computed as the expected value from Bayesian network models by summing the mid-point value of each state weighted by its 
Froese et al., Moving window analysis links landscape-scale resource utilization to habitat suitability models of feral pigs in northern Australia

probability of occurrence. Accordingly, $x_{r}$ could potentially range between 10 and 90 (100\% probability that the requirement is in a very poor (mid-point value 10) and very good (mid-point value 90) state respectively).

For each requirement, we also computed different landscape values $y_{r}$, which described its sufficiency to sustain breeding in feral pigs. Requirement sufficiency was equally discretised into five states corresponding to numerical value ranges from 0-20 to 80-100. For each $r$, we elicited two response functions $f_{r}$ from individual experts $(\mathrm{n}=6)$ that described distance-dependent $\left(f_{D r}\right)$ and composition-dependent $\left(f_{C r}\right)$ relationships between landscape-scale patterns of $x_{r}$ and $y_{r}$. Experts defined $f_{r}$ in five distance/abundance categories and according to the five discrete states of $y_{r}$ (in what sufficiency range is $y_{r}$, if $x_{r}$ falls within a certain distance range/ if there is a certain abundance range of $x_{r}$ accessible?), resulting in step-wise response curves. Each expert's $f_{r}$ were used to parameterize moving window size and analysis metric and compute distance-dependent, composition-dependent and combined distance/composition-dependent requirement landscape values $\left(y_{D r}, y_{C r}\right.$ and $\left.y_{D C r}\right)$. All analyses used circular-shaped moving windows and were implemented in the $R$ 'raster' package (R Core Team 2015, Hijmans 2015) and ESRI ArcGIS 10.2 software.

$y_{D r}$ was computed as the distance-weighted maximum of all pixel values $x_{r i}$ within a neighbourhood of $i=$ $1, \ldots, n$ pixels around a focal pixel (1). Weights $\mathrm{w}_{r i}$ were assigned according to response function $f_{D r}$ and ranged between 0.1 (zero weight was reserved to $x_{r}$ outside the moving window) and 1 (no distance penalty).

$$
y_{D r}=\max f_{D r}\left(x_{r i} \times w_{r i}\right), \quad x=\{10, \ldots, 90\}, \quad i=\{1, \ldots, n\}, \quad w=\{0.1, \ldots, 1\}
$$

$y_{C r}$ was computed by applying function $f_{C r}$ to the mean value $\bar{x}_{r}$ of all pixel values $x_{r i}$ within a neighbourhood of $i=1, \ldots n$ pixels around a focal pixel (2), normalized to the effective value range $\bar{x}_{\text {rmin }}$ to $\bar{x}_{\text {rmax }}$.

$$
y_{C r}=f_{C r}\left(\bar{x}_{r}\right), \quad \text { where } \bar{x}_{r}=\frac{\sum_{i=1}^{n} x_{r i}}{n}, \quad x=\{10, \ldots, 90\}, \quad i=\{1, \ldots, n\}
$$

$y_{D C r}$ was computed in a similar way to $y_{C r}$ by applying function $f_{C r}$ to the normalized mean value $\bar{x}_{r}$. However, pixel values $x_{r i}$ contributing to $\bar{x}_{r}$ were also distance-weighted with $\mathrm{w}_{r i}$ according to response function $f_{D r}(3)$.

$$
y_{D C r}=f_{D C r}\left(\bar{x}_{r}\right), \quad \text { where } \bar{x}_{r}=\frac{\sum_{i=1}^{n} x_{r i} \times w_{r i}}{n}, \quad x=\{10, \ldots, 90\}, \quad i=\{1, \ldots, n\}
$$

Here, we do not describe which landscape values $y_{r}$ were selected and how they were combined to compute a habitat suitability index. Instead, we schematically illustrate how various assumptions about the characteristic scale and mechanism of resource utilization influenced computed sufficiency $y_{r}$. For this purpose we generated a hypothetical distribution of high and moderate quality resources $x_{r}$ - arranged as a large continuous patch, scattered moderate-sized patches, scattered small patches and an isolated small patch - on a $20 \times 20$ pixel grid (Figure 2 top panel). Mirroring our modelling approach, we then applied moving window analysis on circular windows of different sizes (radius $=1,3$ or 5 pixels) and assuming a linear decay of response functions $f_{D r}$ and $f_{C r}$ and investigated the effect of analysis parameters on computed $y_{D r}, y_{C r}$ and $y_{D C r}$.

\section{RESULTS}

Experts identified four key habitat requirements as influential to habitat suitability: water and food resources as well as protection from heat stress and disturbance. Expert-elicited distance-dependent and compositiondependent response functions for each of these requirements are shown in Figure 1. Three general shapes of response curve were described: linear decay, where the sufficiency of a requirement diminishes steadily with increasing distance/decreasing abundance, exponential decay where sufficiency is strongly affected by initial increases in distance or decreases in abundance, and inverted exponential decay where sufficiency diminishes little initially but strongly at large distances or low levels of abundance. Assumptions about the characteristic scale of the response, and hence the slope of the distance-dependent function, varied among experts. The maximum distance beyond which resources become inaccessible (i.e. of no value) was estimated at 1, 2 or 3 $\mathrm{km}$, which corresponded to assumed circular home range sizes of $3.17,12.57$ and $28.51 \mathrm{~km}^{2}$ respectively.

Experts concurred strongly in their description of some responses, e.g. the composition-dependent response to $\bar{x}_{\text {Water }}$ always displayed inverted exponential decay, the distance-dependent response to $x_{\text {Disturbance }}$ followed an exponential decay shape and the distance-dependent response to $x_{F o o d}$ was described by most experts as a linear function, albeit with different slopes. However, experts also showed considerable disagreement on other responses such as the distance-dependent response to $x_{\text {Water }}$ or $x_{\text {Heat }}$. Average curve shapes (dashed pink lines in Figure 1) were near linear for distance-dependent responses to most requirements except $x_{\text {Disturbance }}$ and followed an inverted exponential pattern for composition-dependent responses, although $y_{C F o o d}$ and $y_{C D i s t u r b a n c e}$ diminished much more steadily with decreasing abundance. 
Froese et al., Moving window analysis links landscape-scale resource utilization to habitat suitability models of feral pigs in northern Australia
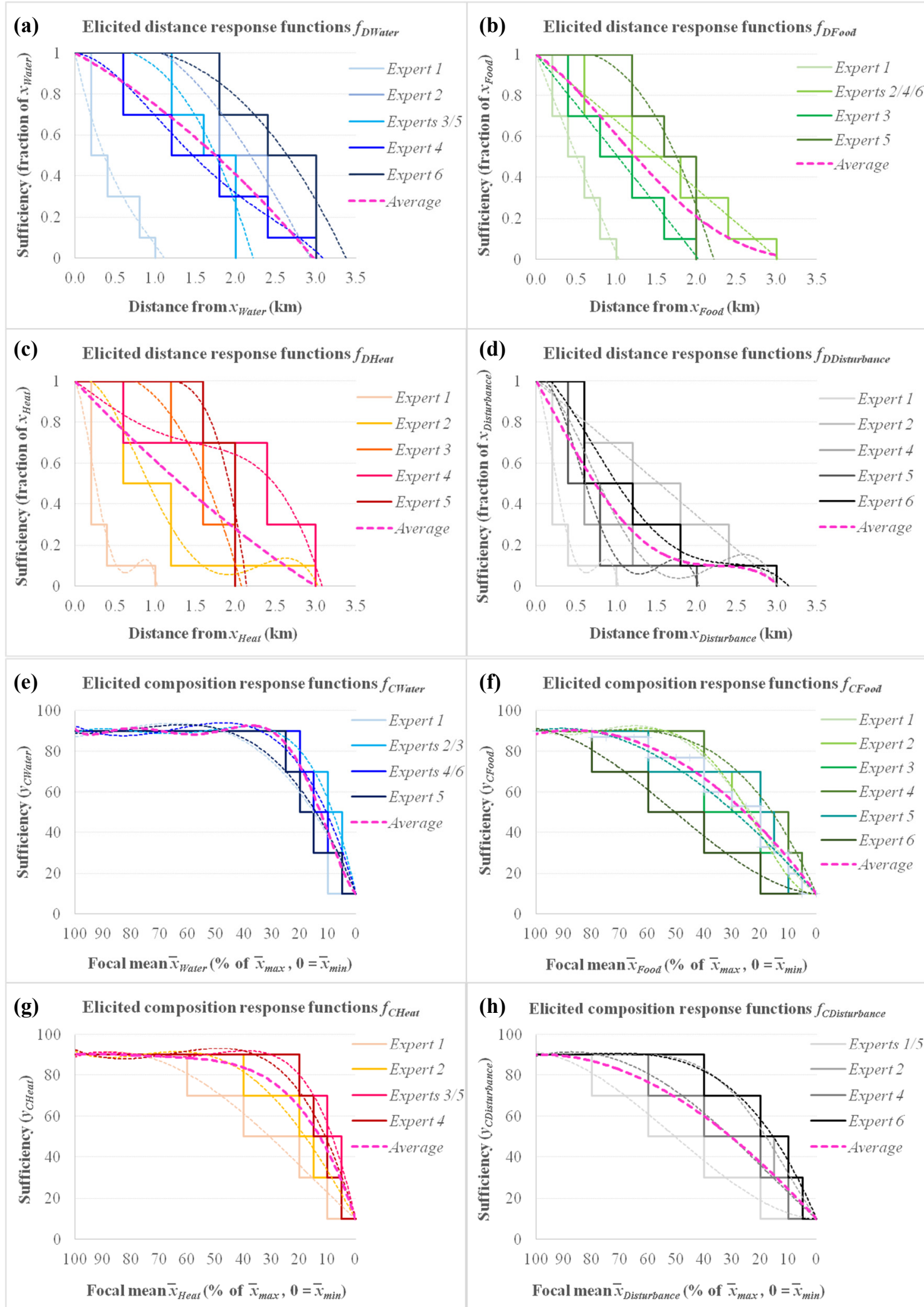

(h) Elicited composition response functions $f_{\text {CDistursance }}$

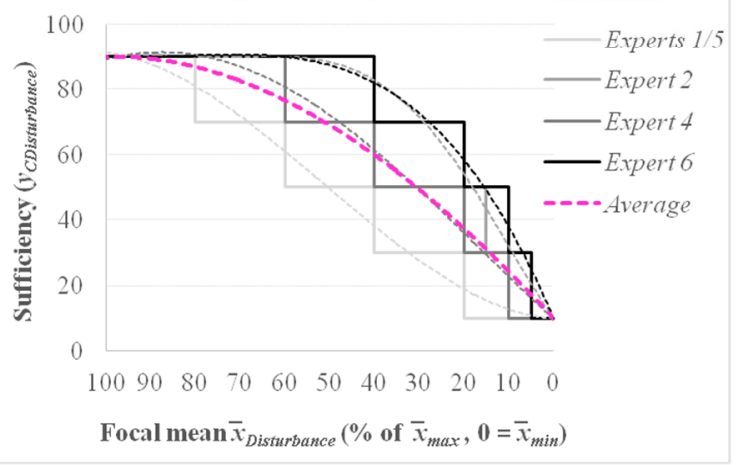

Figure 1: Expert-elicited response functions for the four key habitat requirements of feral pigs: water, food, protection from heat and protection from disturbance. Panels (a) to (d) show diminishing sufficiency of each requirement with increasing distance. Curves cross the $\mathrm{x}$ axis in different places because individual experts had different assumptions about the maximum distance beyond which a resource becomes inaccessible, i.e. of no value to feral pigs. Panels (e) to (h) show diminishing sufficiency of each requirement with decreasing abundance. As elicited response curves followed a step-wise pattern (solid lines), polynomial trend lines are also shown for each expert (dashed lines) as well as the average of all experts (pink dashed line). 
Froese et al., Moving window analysis links landscape-scale resource utilization to habitat suitability models of feral pigs in northern Australia

The effect of various expert assumptions about the characteristic scale and mechanism of resource utilization on computed sufficiency $y_{r}$ is illustrated in Figure 2. Assuming distance-dependence $\left(y_{D r}\right.$, Figure 2 first column) dramatically increased computed sufficiency across all hypothetical resource patterns, essentially by letting the value of each mapped resource, however small, influence a broad neighbourhood. This effect became more pronounced when assuming larger characteristic scales/ moving window sizes (Figure 2 middle and bottom rows). Inverted exponential decay of the response would also enhance this effect while exponential decay would weaken it somewhat. Assuming composition- or combined distance/compositiondependence $\left(y_{C r}\right.$ or $y_{D C r}$, Figure 2 third and second column respectively) both produced similar effects. As the moving window size increased, requirement sufficiency was increasingly consolidated to larger, more continuous resource aggregations; these aggregations also influenced computed $y_{C r}$ and $y_{D C r}$ in areas between patches that contain no resources themselves. On the other hand, isolated or scattered small patches of resources were computed as insufficient at moderate and large window sizes respectively (Figure 2 second and third row). All these effects were slightly less pronounced for $y_{D C r}$.

\section{DISCUSSION}

Knowledge of the characteristic scale and mechanism of resource utilization by feral pigs is limited to few localised studies (e.g. Choquenot \& Ruscoe 2003, Dexter 1998). Yet, this information is needed to account for species mobility and home range behaviour in habitat suitability models. Our results demonstrate that such knowledge can be elicited from experts with field knowledge of feral pigs' response to habitat conditions. Experts were in agreement over several responses. For example, all concurred that even small amounts of water or heat refuge within an accessible neighbourhood are sufficient to sustain breeding in feral pigs up to a threshold where sufficiency rapidly diminishes. Conversely, faced with disturbance pressure from control activities, all experts suggested that the sufficiency of disturbance refuge diminishes rapidly even at short distances. The landscape-scale sufficiency of food resources to feral pig mobs steadily increased as resources became more abundant or into closer proximity. Other responses, e.g. to the distance of the closest accessible water resource or heat refuge, were described very differently by each expert. Such disagreement may be due to knowledge gaps or to actual differences in feral pig behaviour as observed by experts in their respective regions of expertise. In particular, characteristic scales at which feral pigs respond to habitat conditions may vary considerably between regions, for example due to the effect of daytime temperatures on mobility. In another study, experts from southern Queensland defined larger characteristic scales for all requirements, especially during the colder winter months (radius $=5 \mathrm{~km}$, Murray et al. 2015).

Disagreement on the shapes and slopes of response curves aside, the main question concerns the underlying mechanism of feral pigs' response to each habitat requirement, i.e. is landscape-scale utilization mainly distance-, composition- or configuration-dependent? Using a hypothetical arrangement of resources, we showed that moving window analysis metrics (representing the response mechanism) and window sizes (representing the characteristic scale of the response) had a considerable influence on computed requirement sufficiency $y_{r}$. The effects of distance-dependence corresponded well to the response of feral pigs to water resources or heat refuge suggested by experts during elicitation. The effects of composition- or combined distance/composition-dependence reflected the described relationship between landscape-scale patterns of food resources and their sufficiency to sustain feral pig breeding. This suggests that feral pigs may respond to different habitat requirements at different characteristic scales and according to different underlying mechanisms. Moving window analysis should be parameterized for each requirement independently. Lastly, as composition- or combined distance/composition-dependence produced similar effects, the latter should be preferred as it is arguably ecologically more meaningful (the closer a resource, the larger its value).

Clearly, when using expert assumptions about feral pigs' landscape-scale utilization of resources and other habitat requirements to parameterize habitat suitability models, these assumptions must be validated in terms of their effect on model predictive performance. Here, preliminary results prepared in another manuscript suggest that models which likely overestimate suitable feral pig habitat (e.g. incorporate distance-dependent landscape values measured at large characteristic scales) perform better than more conservative models. However, any model that accounted for species mobility through landscape-scale predictors showed higher validity than a model based solely on pixel-scale predictors. Further research could also test the validity of expert assumptions through field-based studies. In particular, feral pigs' configuration-dependent response to resource patterns proved difficult to elicit from experts and was not analysed here, despite suggestions of its importance. Although $y_{D C}$ is arguably influenced by the arrangement or positioning of resources within the moving window, empirical data is needed to clarify the effect of resource configuration. Finally, we make simplifying assumptions about accessible home ranges, notably that they have a circular shape and fixed size. Further developments in moving window analyses to allow for more realistic, irregular home ranges whose size can vary according to the spatial resource patterns encountered would likely improve results. 
Froese et al., Moving window analysis links landscape-scale resource utilization to habitat suitability models of feral pigs in northern Australia

\section{$\mathrm{x}$, pixel only}
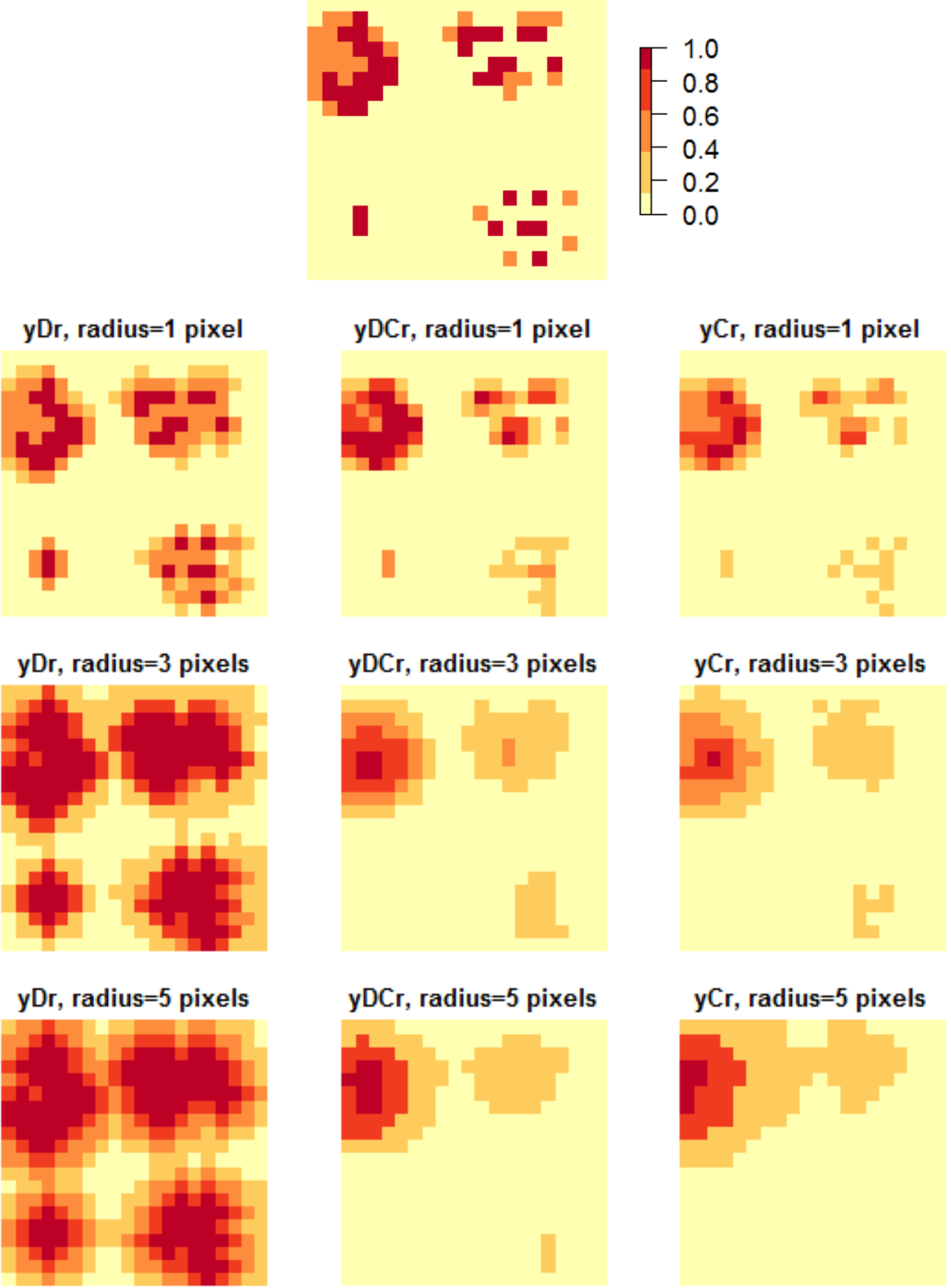

Figure 2: Schematic illustrating the effect of various spatial analysis parameters on computed requirement sufficiency $y_{r}$. Each panel shows a grid of $20 \times 20$ pixels. Requirement pixel values $x_{r}$ prior to spatial analysis (top) take the values 1 (red=high quality), 0.5 (orange=moderate quality) or 0 (yellow=absent). Four spatial patterns of pixel values are illustrated: a continuous large patch (top left corner), scattered moderate-sized patches (top right corner), scattered small patches (bottom right corner) and an isolated small patch (bottom left corner). The 9 panels below show results from moving window analysis on $x_{r}$ to calculate distancedependent ( $y D_{r}$, first column), combined distance/composition-dependent ( $y D C_{r}$, second column) and composition-dependent $\left(y C_{r}\right.$, third column) landscape values. All analysis used circular windows with a radius of 1 pixel (top row), 3 pixels (middle row) and 5 pixels (bottom row) respectively. 
Froese et al., Moving window analysis links landscape-scale resource utilization to habitat suitability models of feral pigs in northern Australia

\section{CONCLUSIONS}

Ecological theory suggests that mobility and home range behaviour must be accounted for when investigating wildlife-habitat relationships. We developed a novel approach for incorporating landscape-scale of resources and other habitat requirements into habitat suitability models of feral pigs via moving window analysis. Here, we presented expert-elicited response functions describing the relationship between landscape-scale patterns of each of four key habitat requirements and their sufficiency to sustain breeding in feral pigs. These functions were used to parameterize moving window analysis. We also schematically illustrated that expert assumptions about the characteristic scale and mechanism of resource utilization had a considerable effect on the computed sufficiency of a requirement. Hence, these assumptions require careful consideration when using them as an input into habitat suitability models. Importantly, feral pigs may respond to different requirements at different scales and according to different underlying mechanisms. Most importantly, the effect of assumptions on model performance must be validated, which will be described in another manuscript by the authors. Our approach is flexible in both spatial extent and data requirements and could readily be applied to other mobile species that respond to habitat conditions at the landscape scale.

\section{ACKNOWLEDGEMENTS}

More than 15 experts of feral pigs in northern Australia were involved in parameterizing the model. Jim Mitchell, Justin Perry, Col Dollery, Andrew Hartwig, Scott Middleton and Tim Kerlin participated in lengthy interviews during which response functions described in this paper were developed. Justin Perry helped with $\mathrm{R}$ analysis. Justine Murray helped with expert elicitation. UQ/CSIRO iNRM scholarship provided funding.

\section{REFERENCES}

Bengsen, A.J., Gentle, M.N., Mitchell, J.L., Pearson, H.E. \& Saunders, G.R. (2014). Impacts and management of wild pigs Sus scrofa in Australia. Mammal Review, 44(2): 135-147.

Burt, W.H. (1943). Territoriality and home range concepts as applied to mammals. Journal of Mammalogy, 24(3): 346-352.

Choquenot, D. \& Ruscoe, W.A. (2003). Landscape complementation and food limitation of large herbivores: habitat-related constraints on the foraging efficiency of wild pigs. Journal of Animal Ecology, 72(1): 14-26.

Cowled, B.D., Giannini, F., Beckett, S.D., Woolnough, A., Barry, S. et al. (2009). Feral pigs: predicting future distributions. Wildlife Research, 36(3): 242-251.

Dexter, N. (1998). The influence of pasture distribution and temperature on habitat selection by feral pigs in a semi-arid environment. Wildlife Research, 25(5): 547-559.

Dijak, W.D., Rittenhouse, C.D., Larson, M.A., Thompson, F.R. \& Millspaugh, J.J. (2007). Landscape habitat suitability index software. The Journal of Wildlife Management, 71(2): 668-670.

Doran, R.J. \& Laffan, S.W. (2005). Simulating the spatial dynamics of foot and mouth disease outbreaks in feral pigs and livestock in Queensland, Australia, using a susceptible-infected-recovered cellular automata model. Preventive Veterinary Medicine, 70(1-2): 133-152.

Guisan, A. \& Thuiller, W. (2005). Predicting species distribution: offering more than simple habitat models. Ecology Letters, 8(9): 993-1009.

Hijmans, R.J. (2015). Package 'raster': geographic data analysis and modeling, URL http://cran.rproject.org/web/packages/raster/.

Holland, J.D., Bert, D.G. \& Fahrig, L. (2004). Determining the spatial scale of species' response to habitat. BioScience, 54(3): 227-233.

Martin, A.E. \& Fahrig, L. (2012). Measuring and selecting scales of effect for landscape predictors in species-habitat models. Ecological Applications, 22(8): 2277-2292.

McGarigal, K. \& Marks, B.J. (1995). FRAGSTATS: spatial pattern analysis program for quantifying landscape structure. General Technical Report PNW-GTR-351, U.S. Department of Agriculture, Forest Service, Pacific Northwest Research Station.

Murray, J.V., Froese, J., Perry, J., Navarro Garcia, J. \& van Klinken, R.D. (2015). Impact modelling for rabbits and feral pigs in QMDB, CSIRO Biosecurity Flagship, Brisbane.

R Core Team (2015). $R$ : a language and environment for statistical computing. R Foundation for Statistical Computing, Vienna, Austria. URL http://www.R-project.org/.

Smith, C.S., Howes, A.L., Price, B. \& McAlpine, C.A. (2007). Using a Bayesian belief network to predict suitable habitat of an endangered mammal: the Julia Creek dunnart (Sminthopsis douglasi). Biological Conservation, 139(3-4): 333-347.

Wiens, J.A. (1989). Spatial scaling in ecology. Functional Ecology, 3(4): 385-397. 